\title{
Vascular Addressins Are Induced on Islet Vessels during Insulitis in Nonobese Diabetic Mice and Are Involved in Lymphoid Cell Binding to Islet Endothelium
}

\author{
Arno Hänninen, * " Cariel Taylor," Philip R. Streeter," Leslie S. Stark," Jaime M. Sarte," Judith A. Shizuru," \\ Olli Simell, ${ }^{\ddagger}$ and Sara A. Michie"l" \\ ${ }^{*}$ National Public Health Institute; ${ }^{\ddagger}$ Department of Pediatrics, University of Turku, 20520 Turku, Finland; ${ }^{\S}$ Department of Internal \\ Medicine and "Department of Pathology, Stanford University School of Medicine, Stanford, California 94305; and "Department of \\ Veterans Affairs, Center for Molecular Biology in Medicine, Palo Alto, California 94304
}

\begin{abstract}
In the nonobese diabetic ( NOD) mouse, lymphocytic and monocytic infiltration of the pancreatic islets leads to beta cell destruction. To investigate the mechanisms by which lymphocytes enter the NOD pancreas, pancreata were immunostained using monoclonal antibodies to a variety of adhesion molecules known to be involved in lymphocyte binding to vascular endothelium, an initial step in the migration of lymphocytes from blood into organized lymphoid and inflamed tissues. These adhesion molecules include: lymphocyte homing receptors involved in tissue-selective binding of lymphocytes to peripheral lymph node (L-selectin) or mucosal lymphoid tissue (LPAM-1, $\alpha_{4} \beta_{7^{-}}$ integrin) high-endothelial venules (HEV); and HEV ligands peripheral vascular addressin (PNAd) and mucosal vascular addressin (MAdCAM-1). In NOD pancreata, $\alpha_{4} \beta_{7}$ is expressed on most infiltrating cells at all stages of insulitis, whereas $\mathbf{L}$-selectin expression is more pronounced on cells in the islets at later stages. During the development of insulitis, MAdCAM-1 and to a lesser extent PNAd became detectable on vascular endothelium adjacent to and within the inflamed islets. The Stamper-Woodruff in vitro assay was used to examine lymphoid cell binding to such vessels. These functional assays show that both the mucosal (MAdCAM-1 / $\alpha_{4} \beta_{7}$ ) and the peripheral (PNAd/L-selectin) recognition systems are involved in this binding. Our findings demonstrate that expression of peripheral and mucosal vascular addressins is induced on endothelium in inflamed islets in NOD pancreas, and that these addressins participate in binding lymphoid cells via their homing receptors. This suggests that these adhesion molecules play a role in the pathogenesis of diabetes in these mice by being involved in the migration of lymphocytes from blood into the inflamed pancreas. (J. Clin. Invest. 1993. 92:2509-2515.) Key words: insulin-dependent diabetes • NOD mouse • adhesion molecules $\bullet$ inflammation $\bullet$ insulitis
\end{abstract}

\section{Introduction}

Extravasation of lymphocytes from the circulation into organized lymphoid tissues and extralymphoid sites is essential in normal immune surveillance as well as in the accumulation of lymphocytes in nonlymphoid tissues during inflammation (1-

Address correspondence to Dr. Sara A. Michie, Department of Veterans Affairs, Center for Molecular Biology in Medicine, 3801 Miranda Avenue, Palo Alto, CA 94304. 1993

Received for publication 7 April 1993 and in revised form 24 June

The Journal of Clinical Investigation, Inc.

Volume 92, November 1993, 2590-2515
$5)$. This extravasation is currently viewed as a multistep process involving recognition and specific interaction of several adhesion molecules on the lymphocyte with their endothelial ligands $(6,7)$. Such pairing of adhesion molecules is necessary to enable the initial binding of the lymphocyte to the endothelial surface, and at each following step leading to lymphocyte migration through the vessel endothelial cell layer into the tissue. Lymphocyte subpopulations use different but overlapping sets of receptors (7), which in physiologic conditions interact only with their corresponding ligands on endothelial cells. Certain endothelial ligands are normally expressed in a tissue-selective manner so that their interaction with lymphocyte adhesion molecules plays a key role in the tissue-selective homing of lymphocytes $(8,9)$. Other endothelial ligands can be expressed in a wide variety of normal or inflamed tissues $(10,11)$. In such situations, these receptors may be used in a non-organ-specific manner to enable rapid recruitment of cells into the tissue.

The mucosal vascular addressin (MAdCAM-1) ${ }^{1}$ and the peripheral vascular addressin (PNAd) are adhesion molecules preferentially expressed on endothelial cells of high-endothelial venules (HEVs) in mucosal organized lymphoid tissues such as Peyer's patches (PP) or on HEVs of peripheral lymph nodes $(\mathrm{PLN})$, respectively $(8,9)$. CD54 (ICAM-1) is a non-organspecific adhesion molecule which is constitutively expressed on endothelium at low levels, but is increasingly expressed on endothelial cells and other cell types after activation by inflammatory mediators (12).

Vascular cell adhesion molecule-1 (VCAM-1) is also found on activated endothelia in a variety of tissues although some expression has been noted on endothelium from histologically normal tissues (13). Antigens expressed on lymphocytes and associated with their homing in a tissue-selective manner to organized lymphoid tissues are known as homing receptors (HR). L-Selectin is a peripheral HR that is essential for lymphocyte homing to PLN but not to mucosal tissues (14). PNAd comprises the surface molecules presenting PLN HEVselective carbohydrate ligands to lymphocyte L-selectin (15). The $\alpha_{4} \beta_{7}$ integrin, previously termed LPAM- 1 or $\alpha_{4} \beta_{\mathrm{p}}$, is a mucosal HR that interacts with MAdCAM-1 $(16,17)$. CD11a/CD18 (LFA-1) is a lymphocyte receptor for CD54. It is present on all leukocytes and has a non-organ-specific function in lymphocyte-endothelial adhesion (18).

To investigate the possible role of adhesion molecules on lymphocytes and vascular endothelial cells in the migration of

1. Abbreviations used in this paper: $\mathrm{HEV}$, high endothelial venule; $\mathrm{HR}$, homing receptor; ICAM-1, intercellular adhesion molecule-1; MAdCAM-1, mucosal vascular addressin; MLN, mesenteric lymph node; PLN, peripheral lymph node; PNAd, peripheral vascular addressin; PP, Peyer's patch; VCAM-1, vascular cell adhesion molecule-1. 
lymphocytes from blood to pancreas and subsequently in the formation of islet infiltrates, we followed the expression of these murine adhesion molecules on inflammatory cells and on endothelium in the pancreases of NOD mice from the age when the mononuclear infiltrates are first detected around islets until the age when advanced insulitis and hyperglycemia occur. The functional status of the adhesion molecules found on lymphocytes and endothelial cells in inflamed islets was determined using an in vitro assay of lymphoid cell-endothelial cell binding.

\section{Methods}

Mice. Mice were examined from two NOD colonies. Both colonies are housed under pathogen-free conditions and fed with normal mouse chow. The Turku colony of NOD mice, bred from animals purchased from Bomholtgård, Ry, Denmark, has a cumulative incidence of diabetes by the age of 25 wk of $~ 80 \%$ in females and $40 \%$ in males. The incidence of diabetes in the Stanford colony is 85\% in females and $45 \%$ in males at 25 wk. Age-matched BALB/c and SJL mice were used as controls.

Monoclonal antibodies. mAbs MECA-367 and MECA-79 (provided by E. Butcher, Stanford University) were used to detect MAdCAM- 1 and PNAd, respectively. MECA-79 reacts with a PNAd carbohydrate-associated epitope, and is a sensitive reagent for immunohistologic assessment of HEV PNAd expression. mAbs MEL-14 (anti-L-selectin), FD441.8 (anti-CD11a), and M18/2.a.8 (anti$\mathrm{CD} 18$ ) were grown in our laboratory from cells obtained from the American Type Culture Collection (Rockville, MD). $\alpha_{4} \beta_{7}$ was detected with mAbs specific for the $\alpha 4$ chain (clone R 1-2 provided by I. Weissman (Stanford University) and also purchased from PharMingen, San Diego, CA; PS / 2 provided by P. Kincade, University of Oklahoma, Norman, $\mathrm{OK}$ ) as well as with $\mathrm{mAbs}$ that react with the $\beta 7$ chain (722, provided by E. Sikorski and E. Butcher, Stanford University; DATK-32, which specifically reacts only with the $\beta 7$ chain of $\alpha_{4} \beta_{7}$ integrin, provided by D. Andrew, Stanford University). MK2.6 (antiVCAM-1) was provided by P. Kincade while 3E2 (anti-CD54) was purchased from PharMingen. The following negative control mAbs were substituted for species- and isotype-matched anti-HR and antiaddressin $\mathrm{mAb}$ in in vitro functional assays and in immunohistochemical stainings: (a) MECA-89 (reacts with an epitope on MAdCAM-1 not involved in lymphocyte binding; used as negative control for MECA367 in the in vitro assays); $(b) 1 \mathrm{G}$ ( reacts with retroviral gp90; negative control for PS/2 and DATK-32); (c) 9B5 (reacts with human L-selectin; negative control for MEL-14); and $(d)$ OZ-42 (reacts with an antigen in developing cerebellum of mouse; negative control for MECA-79).

Immunologic staining of pancreata. Pancreata were taken from NOD and control mice of both sexes at the ages of $3,4,5,6,8$, and 11-12 wk, and from newly diabetic NOD mice and age-matched controls. The pancreata were snap-frozen in liquid nitrogen and were stored at $-70^{\circ} \mathrm{C}$. Serial frozen sections were cut, dried, fixed in cold acetone for $5 \mathrm{~min}$, and stained by indirect immunofluorescence. The sections were first incubated with mAb, followed by FITC-labeled secondary antibody in 5\% normal mouse serum (goat anti-rat IgG; Cappel, Durham, NC; or goat anti-hamster Fab'2, Caltag, South San Francisco, CA for mAb 3E2), next with polyclonal guinea pig anti-porcine insulin (Accurate Chem. \& Sci. Corp., Westbury, NY), and then with Texas red-conjugated goat anti-guinea pig IgG (Accurate Chem.). All incubations were for $30 \mathrm{~min}$ at room temperature followed by three washes in PBS. At least five sections from different levels of a pancreas were stained with each $\mathrm{mAb}$. After staining, sections were mounted in glycerol based medium and viewed by fluorescence microscopy. In some cases, pancreata were immunostained using a three stage immunoperoxidase technique as described, and then were examined by light microscopy (9).

In vitro lymphocyte-endothelial cell binding assay. The StamperWoodruff in vitro binding assay was performed as described but with slight modifications for this study $(19,20)$. Briefly, 8- and 12-wk-old $\mathrm{NOD}$ and BALB/c mice were injected intravenously with $0.2 \mathrm{ml}$ of the intravascular dye Luconyl blue (40\% in saline) (BASF, Ludwigshafen, Germany) and killed 5 min later. NOD pancreata and BALB/c organized lymphoid tissues (PLN and PP) were removed and snap-frozen. Cell suspensions were prepared from BALB/c MLN (mesenteric lymph node) or from one of the following lymphoma cell lines that exhibit tissue-selective binding to HEV in organized lymphoid tissues: $38 \mathrm{C} 13$, a C3H B cell lymphoma that preferentially binds to PLN over mucosal HEV, using an L-selectin/PNAd recognition system $(9,14$, 21 ); or TK1, an AKR T cell lymphoma which expresses $\alpha_{4} \beta_{7}$ but not $\alpha_{4} \beta_{1}$ (VLA-4) and preferentially binds to mucosal over PLN HEV via an $\alpha_{4} \beta_{7} /$ MAdCAM-1 system $(21,22,23)$.

To test the ability of mAbs that react with lymphocyte HR or vascular addressins to interfere with the binding of lymphoid cells to vascular endothelium in inflamed islets in the NOD pancreas, either lymphoid cells or pancreas tissue sections were preincubated with specific mAb, isotype-matched negative control $\mathrm{mAb}$, or media immediately prior to the binding assay as described (19). Tissue sections were then incubated with $1-3 \times 10^{6}$ lymphoid cells in $0.1 \mathrm{ml}$ DMEM for $30 \mathrm{~min}$ at $7^{\circ} \mathrm{C}$ with mild rotation. Nonadherent cells were decanted, and adherent cells were fixed to tissue sections by $1.5 \%$ glutaraldehyde in PBS. Cell binding to vessels was evaluated microscopically under phase or darkfield illumination. Vessels were identified by intravascular localization of Luconyl blue, and their microenvironmental location determined by examination of adjacent sections stained with hematoxylin and eosin or with antiaddressin mAbs using an immunoperoxidase technique. For each pancreas, lymphoid cell binding to vessels in or adjacent to islets was counted only if the vessel could be evaluated on serial sections from the assay treated with specific $\mathrm{mAb}$, negative control mAb, and media. The mean number of cells bound per vessel for each $\mathrm{mAb}$ was determined for pancreas and organized lymphoid tissues from each mouse. These results for each animal are presented as a percentage of media control.

\section{Results}

Non-organ-specific adhesion molecules on inflammatory cells and endothelium. There was strong uniform expression of CD1 1a/CD18 (LFA-1) and CD54 (ICAM-1) by all mononuclear cells that had infiltrated the NOD pancreas. CD54 was also expressed on endothelium of pancreatic vessels in young healthy NOD mice and mice of control strains as well as in prediabetic and diabetic NOD mice. During the development of disease in NOD mice, no conspicious increase in intensity of CD54 expression on endothelial cells was observed (data not shown). Endocrine cells themselves were negative for CD54. VCAM-1 was expressed on endothelium in a subset of pancreatic vessels in BALB/c and NOD mice. In both strains, most $(\geq 97 \%)$ of the VCAM-1 expressing vessels were in the exocrine pancreas. These ranged in appearance from small, flatwalled vessels scattered among the acini to larger veins and arteries adjacent to the interlobular ducts. Occasional small vessels adjacent to uninflamed islets in NOD and BALB/c mice were VCAM-1 positive as were a few vessels within NOD inflamed islets.

Induction of vascular addressins on islet endothelium. There was no expression of PNAd by endothelium in pancreata of control mice (BALB/c and SJL) or in noninflamed pancreata from young, healthy NOD mice. In contrast, a low constitutive expression of MAdCAM-1 was observed on endothelial cells of a few small, flat-walled vessels scattered throughout the exocrine pancreas in all mice (Fig. $1 A$ ), but not on endothelium within the islets.

During the development of islet infiltrates, MAdCAM-1 and PNAd became expressed on islet endothelium. These ad- 

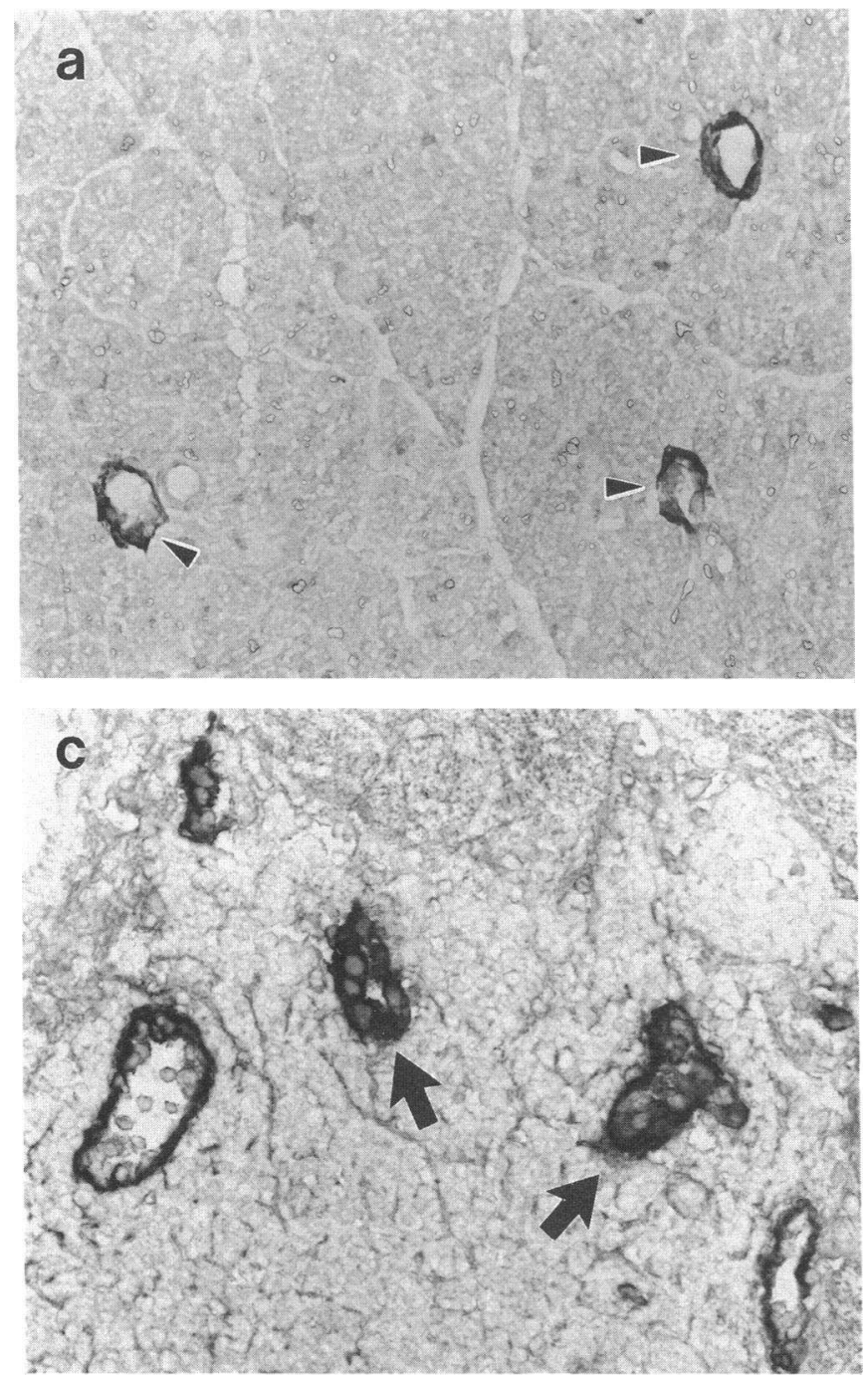

dressins, first detected on endothelium within and adjacent to islets in 4-5-wk-old NOD mice, appeared simultaneously with mononuclear cell infiltrates of these islets. As the degree of insulitis became more severe, small vessels expressing these molecules became more numerous in islets (Fig. $1, b$ and $c$ ). In addition, some of these vessels developed high endothelial morphology and stained with antiaddressin $\mathrm{mAb}$ with an intensity similar to that of HEV in organized lymphoid tissues. Without exception, addressin expression within the islets was limited to vessels that were adjacent to or surrounded by inflammatory cells. In addition, MAdCAM-1 was expressed in the periphery of some islets with periislet infiltration (periinsulitis), but then its intensity was weak. The overall expression of MAdCAM-1 was similar to PNAd within infiltrated islets of $12 \mathrm{wk}$ and older NOD mice, with many vessels coexpressing both addressins. In younger NOD mice, however, more islets vessels expressed MAdCAM-1 than PNAd (Table I). No significant differences in addressin expression were found between pancreata of female and male NOD mice. For example, among the 8-wk-old NOD mice from the Stanford colony (Table I), the percentage of islets expressing MAdCAM-1 or PNAd was 40 or $24 \%$ in the females, and 44 or $29 \%$, respectively in the males.

Expression of $H R$ on infiltrating lymphocytes. The expression of peripheral (L-selectin) and mucosal $\left(\alpha_{4} \beta_{7}\right) \mathrm{HR}$ by lymphocytes in and around inflamed islets was evaluated. The L-

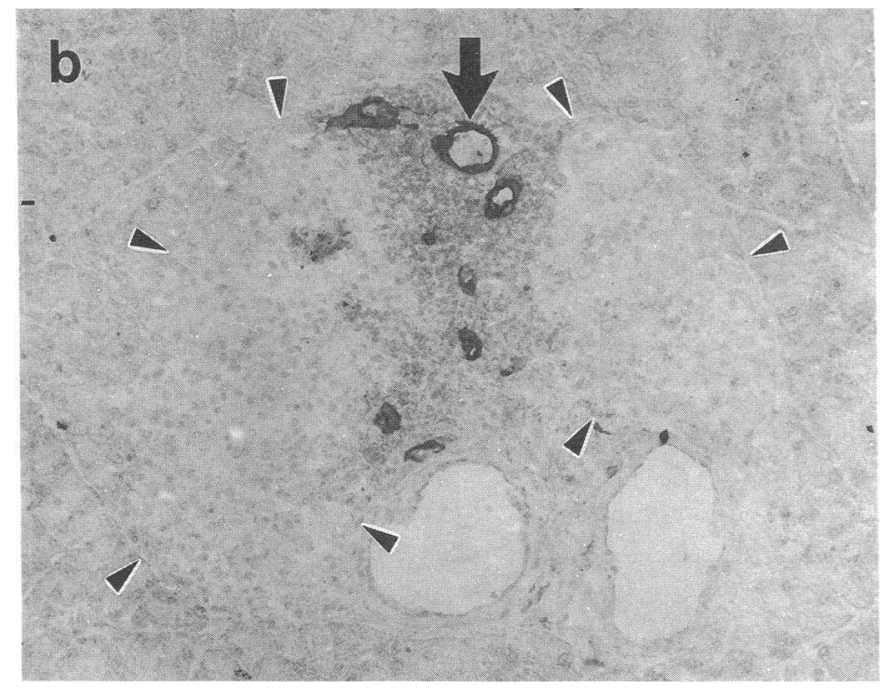

Figure 1. Vascular addressin expression in pancreas. (a) MAdCAM-1 is expressed by vessels (arrowheads) in the exocrine pancreas of a 60-d-old female BALB/c mouse. Similar expression was noted in exocrine pancreas of all SJL and NOD mice examined (immunoperoxidase stain with mAb MECA-367; methylene blue counterstain; $\times 300) .(b)$ Early insulitis in 60-d-old female NOD mouse. Two islets (each outlined by arrowheads) with an intervening lymphocyte infiltrate surrounding several MAdCAM-1-expressing vessels (one vessel highlighted by arrow) (immunoperoxidase stain with mAb MECA$367 ; \times 150)$. (c) Section through an islet with severe insulitis from 90-d-old female NOD mouse. The inflamed islet contains several PNAd-expressing vessels, two of which (arrows) show high endothelial morphology (immunoperoxidase with mAb MECA-79; $\times 400$ ).

selectin peripheral HR was expressed by a minority of peri-and intraislet lymphocytes present during periinsulitis and early insulitis (Fig. $2 a$ ), whereas strong $\alpha_{4} \beta_{7}$ expression as detected by staining with mAbs R1-2 (anti- $\left.\alpha_{4}\right)$ and 722 (anti- $\beta_{7}$ ) was evident on the first cells that gathered in periislet locations as well as on lymphocytes that infiltrated the islets throughout all stages of insulitis (Fig. 2, $b$ and $c$ ). At more advanced stages of insulitis, L-selectin expression intensified among condensed groups of cells within the inflammatory plaque. Hence, the expression of $\mathrm{HR}$ on infiltrating cells shifted from mucosal HR predominance to mixed expression of both HR during progression of insulitis (Table II).

In vitro lymphocyte-endothelial cell binding. The StamperWoodruff in vitro assay was used to study the specific adhesion pathways mediating interactions between lymphoid cells and vascular endothelium of inflamed islets in the NOD pancreas. Our initial assays used lymphocytes obtained from MLN of young adult BALB/c mice. These lymphocytes bound to endothelium of blood vessels in inflamed islets from 8- and 12-wkold female NOD mice. A large portion of this binding could be blocked with mAb directed against MAdCAM-1 and a smaller portion was blocked with mAb against PNAd (Fig. $3 a$ ) ( $t$ test, one-sided: $P=0.0064$ for MECA-367 versus MECA-89; $P$ $=0.038$ for MECA-79 versus OZ-42).

Since MLN is known to contain a mixed population of 
Table I. Expression of Vascular Addressins on Islet Endothelium of NOD-Pancreata during the Development of Insulitis

\begin{tabular}{lccc}
\hline Age of mice $(n)$ & $\begin{array}{c}\text { Islets with } \\
\text { insulitis }\end{array}$ & $\begin{array}{c}\text { Islets with } \\
\text { mucosal addressin }\end{array}$ & $\begin{array}{c}\text { Islets with } \\
\text { peripheral addressin }\end{array}$ \\
\hline & $\%$ & $\%$ & $\%$ \\
4 wk (1) & 6 & 6 & 3 \\
5 wk (2) & $10 \pm 2$ & $7 \pm 5$ & $2 \pm 3$ \\
6 wk (3) & $23 \pm 10$ & $12 \pm 4$ & $5 \pm 9$ \\
8 wk (5) & $25 \pm 16$ & $11 \pm 10$ & $4 \pm 4$ \\
8 wk (4) & $47 \pm 20$ & $42 \pm 13$ & $27 \pm 9$ \\
12 wk (6) & $84 \pm 15$ & $56 \pm 14$ & $51 \pm 26$ \\
12 wk (4) & $81 \pm 10$ & $74 \pm 10$ & $58 \pm 12$ \\
\hline
\end{tabular}

* In each group, four to six pancreata were examined, but only the data from pancreata with inflammatory changes are included in this table. The mean number of islets per pancreas analyzed for MAdCAM-1 and PNAd expression was 26 (range $=10-49$ and range $=11-59$, respectively). ${ }^{\ddagger}$ From Stanford NOD colony. All other mice were from Turku NOD colony.
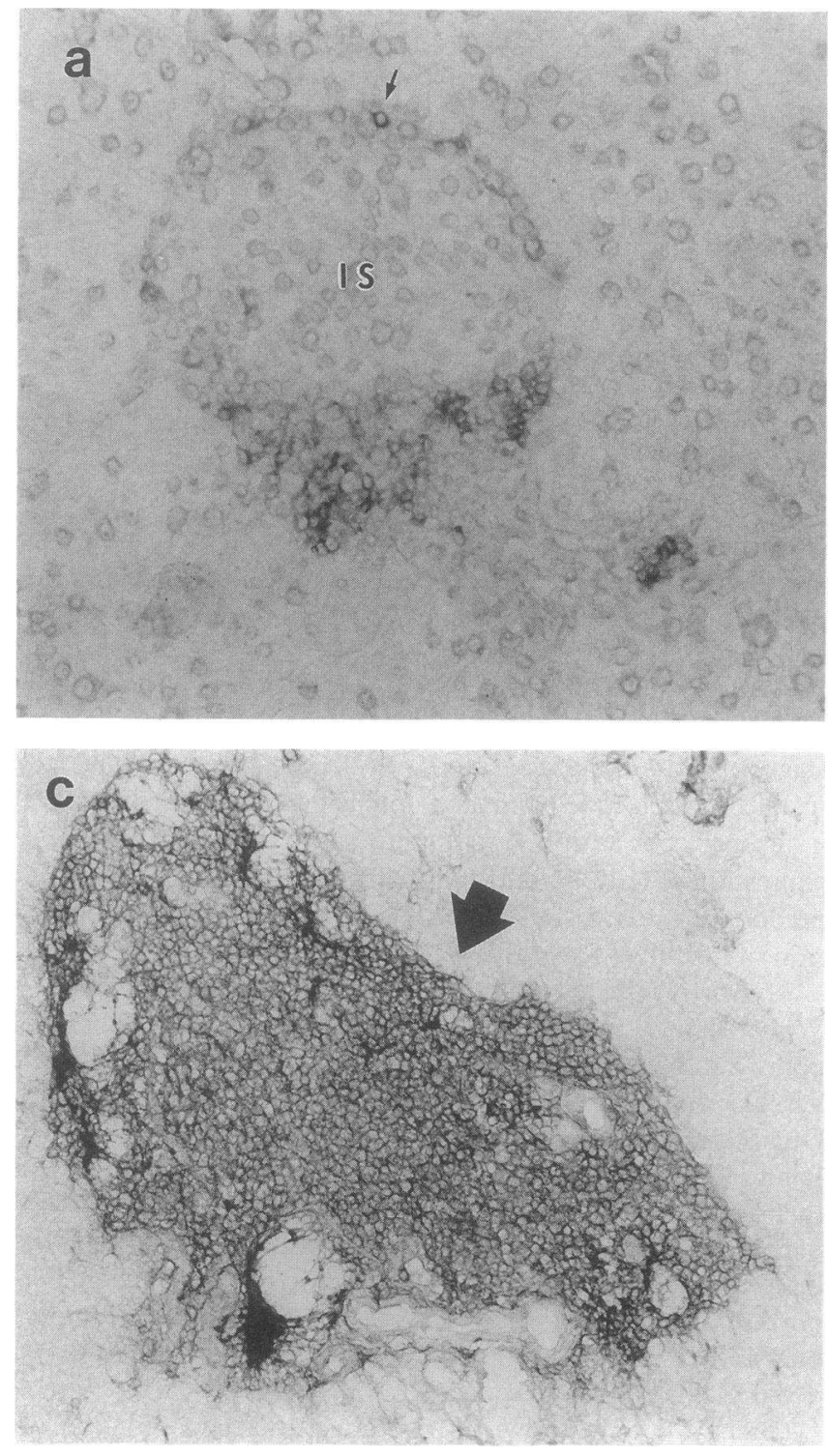

lymphocytes, many of which express both L-selectin and $\alpha_{4} \beta_{7}$ and thus bind to either PNAd or MAdCAM-1-expressing vessels, we chose to use lymphoma cell lines with well-characterized tissue-selective binding properties in subsequent assays. This would allow further dissection of the contributions of the peripheral versus mucosal systems in lymphoid cell binding to endothelium in inflamed islets.

TK1, which binds well to MAdCAM-1-expressing mucosal HEV via its $\alpha_{4} \beta_{7}$ but fails to bind significantly to PLN HEV, bound to many vessels in the inflamed islets of NOD mice. This binding was almost completely inhibited by preincubation of the cells with anti- $\alpha_{4}(\mathrm{mAb} P S / 2)$ or anti- $\beta_{7}(\mathrm{mAb}$ DATK-32), or by preincubation of the pancreas sections with anti-MAdCAM-1 (mAb MECA-367) (Fig. $3 b$ ). 38C13, which binds well to peripheral HEV via L-selectin/PNAd interaction but does not bind significantly to mucosal HEV, also bound to vessels in the inflamed islets. Most of this binding was blocked by mAb directed against PNAd (MECA-79) or L-selectin (MEL-14) (Fig. $3 c$ ). Comparison of the sections from the binding assay with adjacent sections stained with MECA-79 or MECA-367 showed that these lymphoma cells bound to vessels

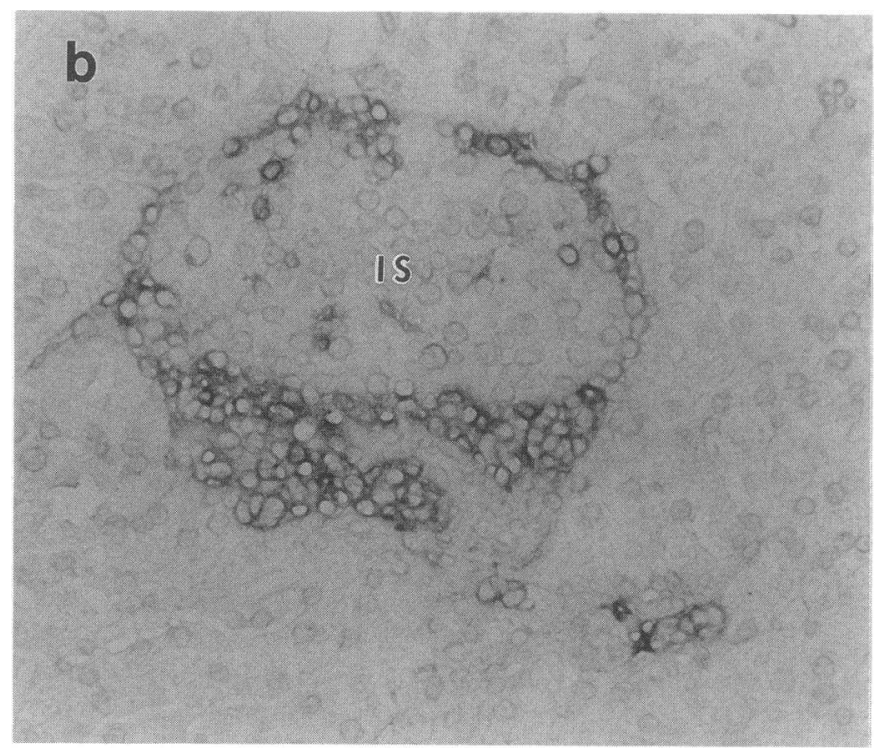

Figure 2. Lymphocyte homing receptor expression in NOD pancreas. $(a$ and $b$ ) Semiserial sections of an islet (IS) from 60-d-old male NOD mouse. There is a lymphocytic infiltrate surrounding the islet with a few lymphocytes within the islet. Some of the lymphocytes express L-selectin (one such lymphocyte is identified by arrow in $a$ ), while most of the lymphocytes express $\alpha_{4}(b)$ and $\beta_{7}$ (not shown) (immunoperoxidase with mAb MEL-14 [a] and R1-2 [b]; 300X). (c) Pancreas from 90-d-old female NOD mouse showing an islet (arrow) completely infiltrated by lymphocytes. The majority of the lymphocytes express $\beta_{7}$ (shown) and $\alpha_{4}$ (not shown) (immunoperoxidase stain with $\mathrm{mAb} 722 ; \times 200)$. 
bearing the appropriate, tissue-specific addressin. Some of these vessels had the histologic appearance of HEVs.

\section{Discussion}

MAdCAM-1 and PNAd are endothelial adhesion molecules in the mouse that are preferentially expressed on HEV in organized lymphoid tissues where they mediate tissue-selective homing of certain subsets of lymphocytes $(8,9)$. In this study, we demonstrate induction of these molecules on islet endothelium during diabetic insulitis in NOD mice. This induction is specific and restricted to endothelial cells within and immediately adjacent to the islets. The initial appearance of these vascular addressins on islet endothelium at the age when insulitis is initiated, the specific localization of addressin-expressing vessels to inflamed (but not uninflamed) islets, and the ability of such vessels to bind lymphocytes in an in vitro assay suggest that these molecules are involved in the regulation of lymphocyte influx into the islets. However, we did not observe strong expression of MAdCAM-1 or PNAd in any islet prior to the

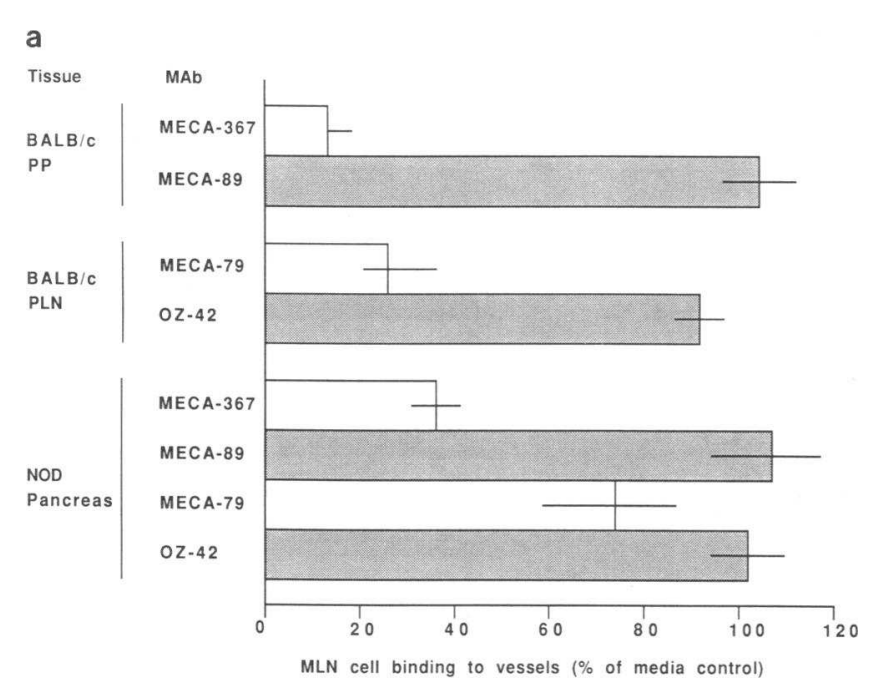

\section{c}

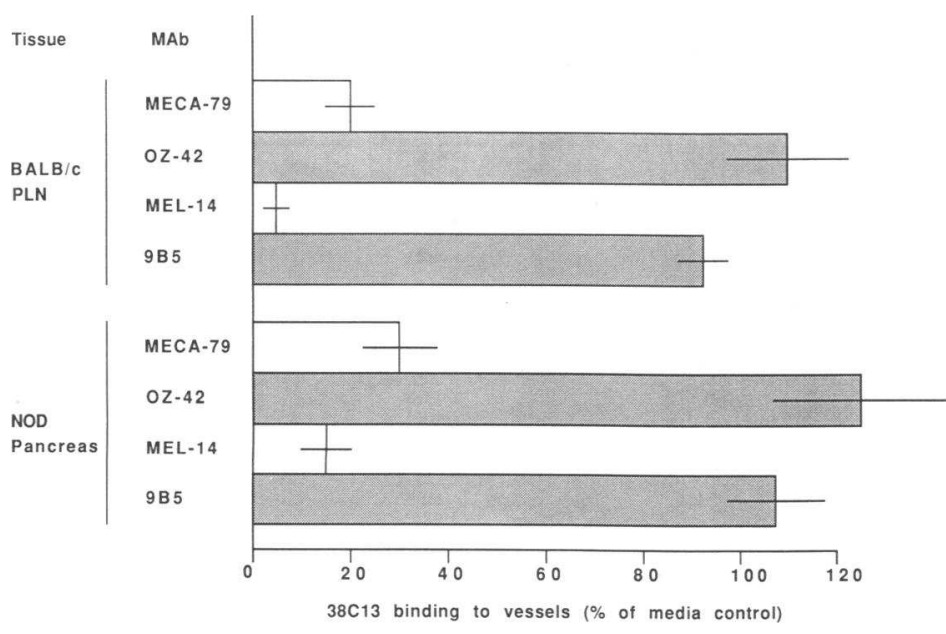

Table II. Expression of a Mucosa-associated $\left(\alpha_{4} \beta_{7}\right)$ and a Peripheral Lymph Node-associated (L-selectin) Homing Receptor on Inflammatory Cells in the Islets of Pancreata* from NOD Mice

\begin{tabular}{lccccc}
\hline & \multicolumn{5}{c}{ Age of mice } \\
\cline { 2 - 6 } & $4 \mathbf{w k}$ & $5 \mathrm{wk}$ & $6 \mathbf{w k}$ & $8 \mathrm{wk}$ & $12 \mathrm{wk}$ \\
\hline LPAM-1 & +++ & +++ & +++ & +++ & +++ \\
L-sel. & + & + & + & ++ & ++ \\
& & & & & \\
\hline
\end{tabular}

* Expression of $\alpha_{4} \beta_{7}$ (staining with mAbs R1-2 and 722) and L-selectin (staining with mAb MEL-14) was evaluated from the same pancreata as in Table I using adjacent sections $(+\leq 33 \%$; $++=34-66 \%$; $+++\geq 67 \%$ of infiltrating cells expressing the HR).

influx of cells. Neither did we observe PNAd expression on vascular endothelium in human nonlymphoid tissues such as skin or thyroid without a coexisting inflammatory infiltrate (41). These data suggest that some other endothelial adhesion

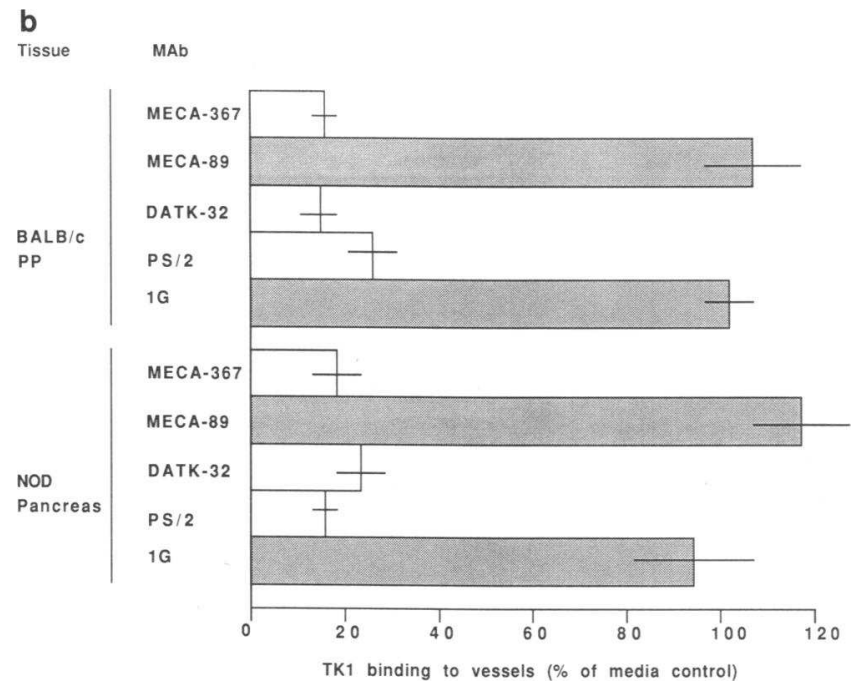

Figure 3. Involvement of homing receptors and vascular addressins in binding of lymphoid cells to endothelium in inflamed islets of NOD pancreata. Each bar represents mean \pm SD of lymphoid cell binding to endothelium on tissue sections from 12-wk-old NOD pancreata $(n=3)$ or BALB/c organized lymphoid tissues $(n=3)$ with addition of anti-HR or anti-addressin $\mathrm{mAb}$ (open bars) or class-matched negative control mAb (shaded bars). Numbers of vessels evaluated per mouse for each mAb treatment ranged from 6 to 17 per pancreas, 7 to 15 per PP, and 17 to 32 per PLN. (a) Pretreatment of tissue sections with MECA-367 (anti-MAdCAM-1) or MECA-79 (anti-PNAd) blocked most of the binding of MLN lymphocytes to HEV in PP and PLN, respectively. These antibodies partially blocked binding of MLN lymphocytes to vessels in inflamed islets. ( $b$ ) As previously described, TK1 cells bound well to HEV in PP but poorly to HEV in PLN (cells/PLN HEV $<6 \%$ of cells/PP HEV; data not shown; see reference 21). The binding to PP HEV was inhibited by $\mathrm{mAb}$ reactive with either chain of the $\alpha_{4} \beta_{7} \mathrm{HR}$ (PS/2, DATK-32) or with MAdCAM-1 (MECA-367). Similar to PP HEV, vessels in inflamed islets bound TK1 cells via an $\alpha_{4} \beta_{7} /$ MAdCAM-1 mucosal recognition system. (c) As previously reported, 38C13 lymphoma cells bound selectively to PLN but not to PP HEV (in this assay, cells/PP HEV < 8\% of cells/PLN HEV; data not shown; see reference 21). The lymphoma binding to PLN HEV and vessels in inflamed NOD pancreas could be inhibited by mAb MEL-14 (anti-L-selectin) or MECA-79 (anti-PNAd). 
molecules could be responsible for initializing lymphocytic infiltration into extralymphoid sites during inflammation and that vascular addressins would be expressed only later on as a consequence of subsequent endothelial activation. Even in that case, the present study suggests that vascular addressins are involved in lymphocyte entry into the islets during sustained islet inflammation, which may be necessary in the pathogenesis of insulin-dependent diabetes. However, in an experimental model of arthritis that is induced by an inflammatory chemical (24), the influx of leukocytes begins simultaneously with activation of endothelial cells showing that in vivo, these phenomena are difficult to observe independently of each other. Accordingly, if expression of addressin molecules on islet-endothelium is increased prior to histologically detectable infiltration of the islets by mononuclear cells, this is a transient stage followed immediately by an influx of inflammatory cells.

The events underlying endothelial activation resulting in induction of endothelial adhesion molecules and increased lymphocyte binding to the endothelium are of prime importance in the accumulation of lymphocytes in inflamed tissues. Not surprisingly, endothelial cells can be activated in vitro by inflammatory mediators (25) and in vivo, by injections of cytokines which cause local changes in endothelial morphology with concomitant influx of cells into tissue $(26,27)$. Within inflamed tissues, these inflammatory mediators can be released by a variety of cells $(4,7)$. First, they may be released from circulating leukocytes coming into contact with endothelium. Second, they may be secreted from endothelial cells themselves. Third, they may be secreted by cells that are normally present in that tissue, including parenchymal cells and resident leukocytes. In the spontaneous autoimmune syndrome of the NOD mouse that results in the accumulation of inflammatory cells in the pancreatic islets, none of these possibilities can so far be excluded. In this context, it is interesting to examine the experiments of Hutchings et al. (28). By administration of a mAb against CD11b/Cd18 that blocks the adhesion of macrophages and monocytes to endothelium, they not only prevented the development of diabetes in NOD mice after cell transfer but also prevented most of the influx of lymphoid cells into islets. This suggests that macrophages may be directly or indirectly involved in the activation of islet endothelium, which then allows the binding and subsequent extravasation of lymphocytes.

MAdCAM-1 was expressed by some vessels in the exocrine pancreas from all NOD, BALB/c, and SJL mice examined, and was the predominant addressin expressed by NOD islet endothelium at early stages of insulitis. This molecule is expressed by endothelium in mucosal tissues such as Peyer's patches and small intestine lamina propria and is the vascular addressin specifically recognized by the $\alpha_{4} \beta_{7} \mathrm{HR}$ found on lymphocytes that home to mucosal sites (17). During embryogenesis, the pancreas develops in mesoduodenum, which is derived from the foregut (29). Hence, it is possible that pancreatic endothelium would differentiate during embryogenesis along with the endothelium in mucosal lymphoid tissues. Alternatively, common environmental factors shared between the adult gut and pancreas might be involved in the expression of MAdCAM-1 by endothelium in both organs. In another study (42), a lymphoid cell line that originated from islet-infiltrates of pancreas from a diabetic human was found to adhere strongly to mucosal HEV, whereas adherence to PLN HEV was much weaker. In the present study, we found that lym- phoid cells infiltrating islets expressed $\alpha_{4} \beta_{7}$ from early on, whereas the expression of L-selectin was initially low and increased with time. These findings suggest that normal pancreas as well as pancreas with early insulitis are predominantly involved in the mucosal lymphocyte recirculation pathway. Alternatively, the small number of cells expressing L-selectin among the cells infiltrating the islets in early insulitis may indicate that the cells are activated (30). This would be consistent with the idea that initially, islets contain many activated cells, either as a result of selective homing of activated cells to the islet or activation of resting lymphocytes once they have entered the islets. The subsequent increase in L-selectin-expressing lymphocytes could be the result of selective migration of L-selectin-expressing cells, or could be due to reexpression of L-selectin on previously activated lymphocytes. Although monocytes can account for some of the L-selectin and $\alpha_{4} \beta_{7}$ integrin expression that was observed, our observations must also reflect HR expression by lymphocytes, since most studies have shown lymphocytes predominate in islet infiltrates $(31,32)$, as was also confirmed by immunohistochemical assessment of leukocytes in pancreata of our mice (data not shown).

In the adhesion cascade leading to extravasation, integrin molecules have an essential role in strengthening the initial adhesion that is often mediated by selectins $(7,33)$. In an inherited disease (leukocyte adhesion deficiency), patients are unable to generate acute inflammatory responses in tissues because of a genetic error in CD18, resulting in severe impairment of leukocyte extravasation (34). Therefore, the uniform expression of this molecule on inflammatory cells in islet-infiltrates is not unexpected. The expression of CD54 on all inflammatory cells in the NOD pancreas, however, contrasts to that seen in human diabetic pancreas, where the expression of CD54 seems to be restricted mainly to endothelial cells (35). In further contrast to the pancreas from a diabetic human, no conspicuous differences in CD54 expression on endothelial cells in nondiabetic and diabetic pancreata could be observed. This may reflect differences in local levels of cytokines such as IL-1, TNF, and $\gamma$-interferon that induce expression of CD54 on many cell types, or differences in inducibility of CD54 expression in different species (36), or simply an observation bias resulting from colocalization of CD54 on inflammatory cells adjacent to endothelium in inflamed islets. CD54 expression on inflammatory cells may be involved in the pathogenesis of insulitis and beta cell destruction in NOD mice, because it may strengthen adhesion during antigen presentation (37), and as a ligand for CD18, it may mediate adhesion between inflammatory cells (38). This may allow the cells to firmly adhere to and interact with each other after extravasation. Recently, injection of anti-CD54 mAb into young NOD mice was shown to reduce the incidence of diabetes significantly (39). Consistent with another previous study (40), we did not detect CD54 expression on insulin positive cells, suggesting that the effect of this treatment was not conferred by blocking any antigen presenting function of beta cells.

In summary, we have shown there is specific induction of mucosal (MAdCAM-1) and peripheral (PNAd) vascular addressins on vessels in inflamed islets of NOD mice, and accumulation within the islets of lymphocytes which express the $\alpha_{4} \beta_{7}$ mucosal and L-selectin peripheral HR. We have used functional assays to demonstrate that both the mucosal (MAdCAM-1 $/ \alpha_{4} \beta_{7}$ ) and the peripheral (PNAd/L-selectin) recognition systems are involved in lymphocyte binding to vessels in 
inflamed islets. This is the first demonstration of induction of functional MAdCAM-1 and PNAd in an extralymphoid site of chronic inflammation. Our results suggest that these vascular addressins and lymphocyte HR, which normally govern lymphocyte traffic to mucosal and peripheral lymphoid organs, are involved in lymphocyte traffic to NOD pancreas. Thus, these adhesion molecules may be expected to play an important role in the development of diabetes in these mice.

\section{Acknowledgments}

We wish to thank D. Andrew, P. Bolt, E. Butcher, B. Englehardt, C. G. Fathman, and S. Jalkanen for thoughtful review of this manuscript.

This work was supported by grants from the Foundation for Diabetes Research (Finland), the Pediatric Research Foundation, Turku University Foundation, Orion Corporation Research Foundation, the Department of Veterans Affairs, the National Institutes of Health (AI19957, DK-39959, GM-37734) and Juvenile Diabetes Foundation International.

\section{References}

1. Gowans, J. L., and E. J. Knight. 1964. The route of recirculation of lymphocytes in the rat. Proc. R. Soc. Lond. B. Biol. Sci. 159:257-282.

2. Yednock, T. A., and S. D. Rosen. 1989. Lymphocyte homing. $A d v$. Immunol. 44:313-378.

3. Jalkanen, S., and E. C. Butcher. 1985. In vitro analysis of the homing properties of human lymphocytes: developmental regulation of functional receptors for high-endothelial venules. Blood. 66:577-582.

4. Pober, J. S., and R. S. Cotran. 1990. The role of endothelial cells in inflammation. Transplantation (Baltimore). 50:537-544.

5. Osborn, L. 1990. Leukocyte adhesion to endothelium in inflammation. Cell. 62:3-6.

6. Stoolman, L. M. 1989. Adhesion molecules controlling lymphocyte migration. Cell. 56:907-910.

7. Butcher, E. C. 1991. Leukocyte-endothelial cell recognition: three (or more) steps to specificity and diversity. Cell. 67:1033-1036.

8. Streeter, P. R., E. L. Berg, B. T. N. Rouse, R. F. Bargatze, and E. C. Butcher. 1988. A tissue-specific endothelial cell molecule involved in lymphocyte homing. Nature (Lond.). 331:41-46.

9. Streeter, P. R., B. T. N. Rouse, and E. C. Butcher. 1988. Immunohistologic and functional characterization of a vascular addressin involved in lymphocyte homing into peripheral lymph nodes. J. Cell Biol. 107:1853-1862.

10. Spertini, O., F. W. Luscinskas, G. S. Kansas, J. M. Munro, J. D. Griffin M. A. Gimbrone, and T. F. Tedder. 1991. Leukocyte adhesion molecule-1 (LAM-1, L-selectin) interacts with an inducible endothelial cell ligand to support leukocyte adhesion. J. Immunol. 147:2565-2573.

11. Elices, M. J., L. Osborn, Y. Takada, C. Crouse, S. Luhowskyj, M. E. Hemler, and R. R. Lobb. 1990. VCAM-1 on activated endothelium interacts with the leukocyte integrin VLA-4 at a site distinct from the VLA-4 / fibronectin binding site. Cell. 60:577-584.

12. Springer, T. A. 1990. Adhesion receptors of the immune system. Nature (Lond.). 346:425-434.

13. Page, C., M. Rose, M. Yacoub, and R. Pigott. 1992. Antigenic heterogeneity of vascular endothelium. Am. J. Pathol. 141:673-683.

14. Gallatin, W. M., I. L. Weissman, and E. C. Butcher. 1983. A cell surface molecule involved in organ-specific homing of lymphocytes. Nature (Lond.). 304:30-34.

15. Lasky, L. A., M. S. Singer, D. Dowbenko, Y. Imai, W. J. Henzel, C. Grimley, C. Fennie, N. Gillett, S. R. Watson, and S. D. Rosen. 1992. An endothelial ligand for L-selectin is a novel mucin-like molecule. Cell. 69:927-938.

16. Holzmann, B., B. W. McIntyre, and I. L. Weissman. 1989. Identification of a murine Peyer's patch-specific lymphocyte homing receptor as an integrin molecule with an $\alpha$ chain homologous to human VLA-4 $\alpha$. Cell. 56:37-46.

17. Berlin, C., E. L. Berg, M. J. Briskin, D. P. Andrew, P. J. Kilshaw, B. Holzmann, I. L. Weissman, A. Hamann, and E. C. Butcher. 1993. $\alpha_{4} \beta_{7}$ integrin mediates lymphocyte binding to the mucosal vascular addressin MAdCAM-1. Cell. 74:185-195.

18. Hamann, A., D. Jablonski-Westrich, A. Duijvestijn, E. C. Butcher, H. Baisch, R. Harder, and H. G. Thiele. 1988. Evidence for an accessory role of LFA-1 in lymphocyte-high endothelium interaction during homing. J. Immunol. 140:693-699.
19. Stamper, H. B., Jr., and J. J. Woodruff. 1976. Lymphocyte homing into lymph nodes. In vitro demonstration of the selective affinity of recirculating lymphocytes for high-endothelial venules. J. Exp. Med. 144:828-33.

20. Butcher, E. C., R. G. Scollay, and I. L. Weissman. 1979. Lymphocyte adherence to high-endothelial venules: characterization of a modified in vitro assay, and examination of the binding of syngeneic and allogeneic lymphocyte populations. J. Immunol. 123:1996-2003.

21. Bargatze, R. F., P. R. Streeter, and E. C. Butcher. 1990. Expression of low levels of peripheral lymph-node associated vascular addressin in mucosal lymphoid tissues: possible relevance to the dissemination of passaged AKR lymphomas. J. Cell. Biochem. 42:219-227.

22. Butcher, E. C., R. G. Scollay, and I. L. Weissman. 1980. Organ specificity of lymphocyte interaction with organ-specific determinants on high-endothelial venules. Eur. J. Immunol. 10:556-561.

23. Ruegg, C., A. A. Postigo, E. E. Sikorski, E. C. Butcher, R. Pytela, and D. J. Erle. 1992. Role of integrin $\alpha_{4} \beta_{7} / \alpha_{4} \beta_{\mathrm{p}}$ in lymphocyte adherence to fibronectin and VCAM-1 and in homotypic cell clustering. J. Cell Biol. 117:179-189.

24. Graham, P. C., and S. L. Shaman. 1972. Peroxidase arthritis. II. Lymphoid cell-endothelial interactions during a developing immunologic inflammatory response. Am. J. Pathol. 69:7-16.

25. Luscinskas, F. W., A. F. Brock, M. A. Arnaout, and M. A. Gimbrone. 1989. Endothelial leukocyte adhesion molecule-1-dependent and leukocyte (CD11/CD18)-dependent mechanisms contribute to polymorphonuclear leukocyte adhesion to cytokine-activated human vascular endothelium. J. Immunol. 142:2257-2263.

26. Colditz, I., R. Zwahlen, B. Dewald, and M. Baggiolini. 1989. In vivo inflammatory activity of neutrophil activating factor, a novel chemotactic peptide derived from human monocytes. Am. J. Pathol. 134:755-760.

27. Munro, J. M., J. S. Pober, and R. S. Cotran. 1989. Tumor necrosis factor and interferon gamma induce distinct patterns of endothelial activation and associated leukocyte accumulation in skin of Pabio anubis. Am. J. Pathol. 135:121133.

28. Hutchings, P., H. Rosen, L. O'Reilly, E. Simpson, S. Gordon, and A. Cooke. 1990. Transfer of diabetes in mice prevented by blockade of adhesionpromoting receptor on macrophages. Nature (Lond.). 348:639-642.

29. Hamilton, W. J., and R. M. H. McMinn. 1976. Digestive system. In Textbook of Human Anatomy. W. J. Hamilton, editor. Macmillan Press, Ltd., London Basingstoke. 329-404.

30. Jung, T. M., W. M. Gallatin, I. L. Weissman, and M. O. Dailey. 1988 Down-regulation of homing receptors after $\mathrm{T}$ cell activation. J. Immunol. 141:4110-4117.

31. Miyazaki, A., T. Hanafusa, K. Yamada, J. Miyagawa, H. Fujino-Kurihara, H. Nakajima, K. Nonaka, and S. Tauri. 1985. Predominance of T lymphocytes in pancreatic islets and spleen of prediabetic non-obese diabetic (NOD) mice: a longitudinal study. Clin. Exp. Immunol. 60:622-630.

32. Shimizu, J., S. Ikehara, J. Toki, H. Ohtsuki, R. Yasumizu, and Y. Hamashima. 1987. Immunohistochemical and ultrastructural studies on beta cell destruction in NOD mouse, an animal model for Type 1 diabetes mellitus. Acta Histochem. Cytochem. 20:9-19.

33. Hynes, R. O. 1992. Integrins: versatility, modulation and signaling in cell adhesion. Cell. 89:11-25.

34. Kishimoto, T. K., R. S. Larson, A. L. Corbi, M. L. Dustin, D. E. Staunton, and T. A. Springer. 1989. The leukocyte integrins. Adv. Immunol. 46:149-182.

35. Hänninen, A., S. Jalkanen, M. Salmi, S. Toikkanen, G. Nikolakaros, and O. Simell. 1992. Macrophages, T cell receptor usage and endothelial cell activation in the pancreas at the onset of insulin-dependent diabetes mellitus. J. Clin. Invest. 90:1901-1910.

36. Dustin, M. L., D. E. Staunton, and T. A. Springer. 1988. Supergene families meet in the immune system. Immunol. Today. 9:213-215.

37. Altmann, D. M., N. Hogg, J. Trowsdale, and D. Wilkinson. Cotransfection of ICAM-1 and HLA DR reconstitutes human antigen presenting cell function in mouse L cells. Nature (Lond.). 338:512-514.

38. Rothlein, R., M. L. Dustin, S. D. Marlin, and T. A. Springer. 1986. A human intercellular adhesion molecule (ICAM-1) distinct from LFA-1. J. Immunol. 137:1270-1274.

39. Amano, K., R. Yoneda, Y. Tominaga, Y. Hirao, T. Taki, and K. Yokono. 1992. Association of ICAM-1 and LFA-1 molecules in the development of diabetes in NOD mice. Diabetes. 41:38a. (Abstr.)

40. O'Reilly, L. A., P. R. Hutchings, P. R. Crocker, E. Simpson, T. Lund, D. Kioussis, F. Takei, J. Baird, and A. Cooke. 1991. Characterization of pancreatic islet cell infiltrates in NOD mice: effect of cell transfer and transgene expression. Eur. J. Immunol. 21:1171-1180.

41. Michie, S. A., P. R. Streeter, P. A. Bolt, E. C. Butcher, and L. J. Picker. 1994. The human peripheral lymph node vascular addressin: an inducible endothelial antigen involved in lymphocyte homing. Am J. Pathol. In press.

42. Hänninen. A. M. Salmi, O. Simell, and S. Jalkanen. 1994. Endothelial cell-binding properties of lymphocytes infiltrated into human diabetic pancreas. Implications for the pathogenesis of IDDM. Diabetes. In press. 